\title{
La francophonie aristocratique russe au XIXe siècle dans les journaux intimes féminins
}

\author{
Michèle Debrenne ${ }^{1, *}$ et Tatiana Makarova ${ }^{2}$ \\ ${ }^{1}$ Université d'Etat de Novossibirsk, 630090 Novossibirsk, Russie \\ ${ }^{2}$ Université d'Etat de Novossibirsk, 630090 Novossibirsk, Russie
}

\begin{abstract}
Résumé. L'article analyse le niveau de maitrise du français tel qu'il est manifesté dans les cahiers intimes d'une princesse russe, écrits en français entre 1830 et 1848. Nous analyserons tout d'abord les marques transcodiques typiques du code switching et leur fonctionnement dans le texte, puis les erreurs de langage relevées dans les 5 cahiers analysés, qui représentent plus de 1000 pages manuscrites. Nous arriverons à la conclusion que le niveau de langue de la diariste et de son entourage était très élevé, très faiblement marqué par des interférences avec la langue maternelle.
\end{abstract}

\begin{abstract}
French language among Russian aristocracy of the XIXth century in women's diaries. In this paper the level of proficiency in French language is analyzed, as it appears in the diaries of a Russian princess, written in French from 1830 to 1848 . We will study transcoding markers in code switching and the way they are integrated in the text. Then we will analyze speech errors collected in the 5 notebooks, i.e. more than 1000 handwritten pages. We conclude that the level of French language of the author and her environment was very high, with very few interferences with mother tongue.
\end{abstract}

\section{Introduction}

L'étude présentée ici se place dans un projet d'édition critique de carnets intimes rédigés en français d'une princesse russe, Olga I. Orlova-Davydova, née Baryatinsky (1814-1873, infra OIOD). En effet, ainsi qu'en conviennent les spécialistes de la francophonie en Russie des XVIII-XIX E. Grechanaia et C. Viollet, « le vaste corpus de journaux personnels tenus pour la plupart en langue française par des femmes et des jeunes filles de l'aristocratie russe à la fin du XVIIIe siècle et durant les premières décennies du XIXe siècle constitue une source très précieuse bien qu'assez peu exploitée. En effet, leur étude se limite trop souvent à quelques textes publiés dont les auteures sont des figures connues voire célèbres - Natalia Dolgoroukova, Catherine II, la princesse Ekaterina Dachkova, Anna Labzina - et essentiellement sous l'angle de l'auto-représentation» [1]. Nous présenterons ici un corpus formé de 5 cahiers de 250 pages chacun, conservés dans la Bibliothèque Scientifique et

\footnotetext{
* Corresponding author : micheledebrenne@gmail.com
} 
Technique de Novossibirsk, et qui représentent des copies autorisées, annotées et corrigées de carnets intimes conservés pour leur part à Moscou. L'intérêt de ce corpus réside notamment dans le fait qu'il correspond à une volonté de perennisation, un projet quasi éditorial ; par exemple, les deux premiers cahiers sont une compilation des originaux, copiés in extenso mais dans un ordre non chronologique. Cette volonté de publication justifie notre propos, qui est de publier le texte français sous forme de corpus électronique balisé (indiquant notamment les erreurs, ratures, corrections et les différentes rédactions) et sa traduction en russe annotée. Dans le présent article nous considérons ces cahiers comme un document essentiel reflétant la francophonie des aristocrates russes de cette époque qui s'étend de 1830 à 1848 . Pour ce faire nous analyserons plus particulièrement les marques transcodiques et les erreurs.

Il est convenu d'affirmer qu'au XVIIIe et XIXe siècle l'aristocratie russe était francophone, de même que celle d'autres pays européens [2]. Il semble toutefois que la maitrise du français était différente selon les cas : comme on peut le constater dans les textes publiés récemment $[3,4]$, la langue écrite par les locuteurs aristocrates de l'époque n'était pas toujours parfaite. Les textes dont nous disposons, ainsi que, comme nous l'avons constaté, la correspondance particulière de OIOD ou de son époux, Vladimir P. OrlovDavydov, sont, par contre, caractéristiques d'un très bon français. Par ailleurs, le bilinguisme (et même le plurilinguisme) des auteurs y est reflété, ce qui nous permet de juger de la pratique du français dans ces milieux.

\section{Les marques transcodiques}

\subsection{Les éléments de russe dans le texte français}

Nous utiliserons ici le terme de «marque transcodique » pour désigner une alternance épisodique de codes par inclusion dans un texte dans une langue de termes isolés venant d'une autre langue. Les inclusions de mots russes dans le texte français sont essentiellement de deux types :

- mots écrits en caractères cyrilliques (selon l'ancienne orthographe)

- mots russes transcrits en caractères latins

Sur les 5 cahiers, qui représentent un total de 163259 mots, on a relevé 629 exemples de marques transcodiques différentes, soit 1038 en nombre absolu. Dans leur immense majorité il s'agit de langue russe, cependant certaines autres langues peuvent être présentes. Il s'agit alors principalement d'anglais, on trouve également quelques mots épars d'allemand.

\subsubsection{Les types de marques transcodiques}

\section{Toponymes.}

Les carnets intimes étudiés comportent un grand nombre de relations de voyages, tant en Europe qu'en Russie. On trouve des toponymes inscrits en cyrillique essentiellement dans les cahiers écrits en Russie, et ils correspondent pour la plupart à des noms de villages russes, tels, par exemple, ceux de leurs propriétés Усолье (Oussolié, non loin de Jigouli) et Ompada (Otrada, au sud de Moscou);

\section{Anthroponymes.}

Sont écrits en cyrillique un certain nombre de noms de famille russes, notamment ceux dont la transcription en russe est malaisée, par ex.: Подзюнинъ (Podzyunin), Хвощинский (Khvochtchinski), ainsi que des prénoms, qui sont ceux des domestiques, auxquels OIOD 
s'adressait en russe: Гриша (Gricha), Ваня (Vania). On a ainsi supposé que l'auteur écrivait ces noms, ainsi que ceux des serviteurs du culte, en cyrillique parce que c'était la langue dans laquelle elle s'adressait à ces personnes.

Les autres exemples de marques transcodiques en cyrillique recouvrent les cas suivants :

\section{Realia}

Il s'agit tout d'abord de titres de fonction, par ex. Статсъ-Дама (dame d'honneur), et notamment ceux des représentants du clergé russe: дъячокъ (diacre). On y trouve également des realia russes traditionnelles telles изба (isba), баня (bain de vapeur). Cependant on ne peut pas dire que l'auteur applique des règles strictes quant aux realia. Par exemple, les substantifs comme droschky (sorte d'équipage) ou iswochtchik (cocher) sont toujours donnés en transcription; d'autres, tels blini, sont tantôt en cyrillique, tantôt en transcription, sans qu'on puisse déterminer de régularité dans cet emploi. C'est dans cette catégorie qu'on trouve le plus de translittérations, mais elles ne représentent en tout qu'une vingtaine de lexèmes.

La plupart des termes concernant la liturgie orthodoxe est donnée en cyrillique, cependant certains termes religieux non spécifiques sont en français, par ex. messe, communier.

\section{Citations}

Les cas de marqueurs transcodiques plus longs, correspondent :

- soit à des alternances codiques de la diariste, notamment dans les passages de son journal adressés à son mari absent, et qui s'apparentent plus à des lettres qu'à des notes de journal ; Certaines se terminent par des phrases tendres en russe, ce qui semblerait indiquer que dans l'intimité, nos personnages se parlaient russe et non français: Прощай, милейший, душа моей души (Adieu, mon chéri, âme de mon âme)

- soit à des citations de personnages russophones, tels les enfants dans leur petite enfance ou les domestiques : Владимирь Петровичь приехаль (Vladimir Petrovitch est arrivé) ; что Маменька мы теперь будемъ читать? (Maman, et maintenant, qu'est-ce qu'on va lire?).

Cette habitude de citer les interlocuteurs dans leur langue fait apparaitre dans le texte du journal des citations (rares) en d'autres langues, par exemple en ukrainien я насльимовъ якъ ты тоже хохлатка, матушка О.И., посоветуй батюшке В.П. Больно нам трудно жить (on m'a dit que toi aussi tu étais ukrainienne, petite-mère, parle à V.P. [Vladimir Petrovitch, l'époux de OIOD], notre vie est bien dure) ou en anglais: I shall be crucified for all my life. Les passages de la liturgie orthodoxe sont, eux, cités en slavon d'église, qui n'était probablement pas ressenti par les locuteurs de l'époque comme une véritable « langue étrangère » : Бы же ту некий человекъ тридесять и осмь леть иныии въ недузи своемъ (Là se trouvait un homme malade depuis trente-huit ans, St Jean 5 : 6-7).

\section{Références culturelles}

On y trouve des noms de saints, notamment quand, par périodes, OIOD en accompagne la date du jour : Samedi 27 (Іоана Златоуста) (Jean Bouche d'Or) ; des noms de magasins Гостиный дворъ (Gostinnyi dvor, magasin à St Pétersbourg) ou institutions connues.

\section{Intertexte}

La plupart des citations consiste en extraits de textes saints, prières et/ou sermons, ou les titres des ouvrages de ce genre et quelques œuvres littéraires, par ex. Мертвые души (les Ames Mortes).

Le Tableau 1 présente la répartition des marques transcodiques selon les différents cahiers (601-1 - 601-5, les numéros correspondant à leur code d'archive GPNTB). Les chiffres entre parenthèses indiquent le nombre total d'occurrences. 
Tableau 1. Répartition des marqueurs transcodiques par cahier

\begin{tabular}{|l|l|l|l|l|l|l|l|}
\hline № & type de marqueur & $601-1$ & $601-2$ & $601-3$ & $601-4$ & $601-5$ & total \\
\hline 1. & realia & $9(14)$ & $16(25)$ & $64(111)$ & $19(22)$ & $20(40)$ & $\begin{array}{l}128 \\
(212)\end{array}$ \\
\hline 2. & anthroponymes & $\begin{array}{l}26 \\
(46)\end{array}$ & $26(66)$ & $89(195)$ & $22(36)$ & $22(50)$ & $\begin{array}{l}185 \\
(393)\end{array}$ \\
\hline 3. & toponymes & $3(5)$ & $20(23)$ & $77(151)$ & $2(4)$ & $27(40)$ & $\begin{array}{l}129 \\
(223)\end{array}$ \\
\hline 4. & citations & $\begin{array}{l}12 \\
(12)\end{array}$ & $3(3)$ & $22(22)$ & $10(10)$ & $4(4)$ & $51(51)$ \\
\hline 5. & intertexte & $5(4)$ & $3(3)$ & $12(18)$ & $1(1)$ & $4(4)$ & $25(30)$ \\
\hline 6. & $\begin{array}{l}\text { références } \\
\text { culturelles }\end{array}$ & $1(1)$ & $1(1)$ & $10(10)$ & $79(79)$ & 0 & $91(91)$ \\
\hline 7. & translittérations & $4(6)$ & $4(6)$ & $5(11)$ & $6(13)$ & $1(2)$ & $20(38)$ \\
\hline & total & $\begin{array}{l}60 \\
(88)\end{array}$ & $\begin{array}{l}73 \\
(127)\end{array}$ & $\begin{array}{l}279 \\
(518)\end{array}$ & $\begin{array}{l}139 \\
(165)\end{array}$ & $\begin{array}{l}78 \\
(140)\end{array}$ & $\begin{array}{l}629 \\
(1038)\end{array}$ \\
\hline
\end{tabular}

On peut faire les constatations suivantes :

- les marqueurs transcodiques en cyrillique sont réparties de manière inégale dans les cahiers et sont particulièrement fréquents dans le cahier 601-3, rédigé alors que la diariste résidait en Russie, dans sa propriété à la campagne, et communiquait principalement avec des paysans ou des gens simples, donc, probablement, en russe;

- la répartition à l'intérieur des catégories est relativement régulière;

- la quantité réduite de marqueurs dans le cahier 601-5 s'explique par le fait qu'il ne contient que 80 pages écrites en français, le reste étant en russe.

\subsubsection{Fonctionnement des marqueurs transcodiques dans le texte}

Il est intéressant de s'attarder sur les modes d'inclusion des mots russes dans le texte français. On remarque, en effet, que malgré le fait qu'elles soient écrites en alphabet cyrillique, les inclusions de mots russes sont entièrement soumises aux règles du français : ainsi, elles sont immanquablement accompagnées d'un déterminant (article) : ils sont allés à un autre подъездъ (porte d'entrée). La plupart du temps, l'article correspond au genre et au nombre du mot russe: les домашніе et nous deux composaient toute la société (domestiques), même si le genre ne correspond pas à celui de l'équivalent français ; à la часть (au commissariat). Dans le cas de substantifs du genre neutre en russe, l'usage varie : féminin pour lа Волостное правление (direction régionale), masculin dans le cas de аи гумно (aire de battage). Pour un substantif russe commençant par une voyelle, on observe l'emploi de l'article élidé l'изба (isba), l'околииа (orée du village). On peut également rencontrer des articles partitifs : donnе ди збитенъ (sbitène, boisson au miel), dи простокваша (lait caillé).

Les substantifs peuvent être employés au pluriel, mais aucune autre trace de modification flexionnelle ne subsiste, toute la rection exigée par la grammaire russe est remplacée par des prépositions : nous sommes allés aи пчельникъ (rucher) ; еn Тарантасъ (tarantasse, voiture de voyage). Même à l'intérieur de syntagmes nominaux complexes en russe les conjonctions ou prépositions restent en français, par ex. : le Строитель de Давыцова Пустьнь (le supérieur du monastère de Davydova Poustyn); Les Delles Акуловъ, Тартеньевъ еt Шереметьевъ оnt chanté (Akoulov, Tarteniev et Chérémétiev). Sur les 5 cahiers étudiés on ne trouve qu'un seul cas de conjonction de coordination en russe : il y avait un service funèbre pour sa belle-mère et elle en même teтрs, княгинь Марии и Марии (les princesses Marie et Marie). D'ailleurs la confrontation avec l'original 
montre que cette conjonction de coordination est du fait du copiste et non de la diariste, qui avait bien écrit княгинь Марии еt Марии.

Ces constatations s'appliquent de manière égale à tous les types de marques transcodiques énumérées plus haut. Les seules marques textuelles, d'un emploi non systématique, de changement de code sont des majuscules et/ou un soulignement (voir fig. 1).

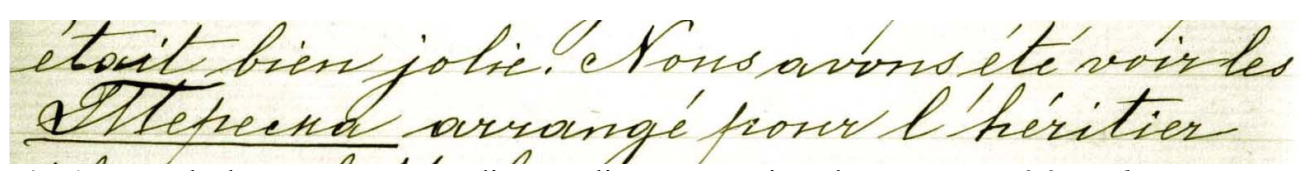

Fig. 1. Exemple de marqueur transcodique souligné avec majuscule Nous avons été voir les mepeмa arrangé pour l'héritier.

Les citations plus longues sont introduites selon les règles du discours direct, cependant l'emploi des guillemets n'est là non plus pas systématique, comme on le voit dans l'exemple suivant de dialogue d'OIOD avec son fils Vladimir : il dit : «какъ бы мне хотелось умереть какъ Товитъ» је діs зачем ? правда иногда так скучна. (il dit: «comme je voudrais mourir comme Tobit» je dis pourquoi ? la vérité est parfois si ennuyeuse. ) où seule la logique de la conversation permet d'attribuer la dernière réplique au jeune garçon.

Ainsi, l'analyse du fonctionnement des marqueurs transcodiques russes dans le texte français des journaux intimes d'OIOD montre une profonde imbrication de ces mots dans le discours, et on peut penser qu'à l'oral le passage d'une langue à l'autre se faisait sans heurt, les mots russes se prononçant à la française avec, par exemple, l'accent tonique sur la pénultième, ce qui n'est pas le cas en russe.

\subsection{Analyse des erreurs dans les journaux intimes de O. I. Orlova-Davydova}

Une des meilleures méthodes d'établissement du portrait linguistique d'une personne, c'està-dire de l'ensemble de ses compétences langagières dans l'une ou l'autre des langues qu'elle pratique, consiste à analyser les erreurs qu'elle fait, en les comparant avec la norme en usage. Dans notre cas il convient, par exemple, de tenir compte des modifications de la norme orthographique dans le cours du XIXe siècle. Les carnets originaux comportent les formes verbales en -ois, les carnets copiés utilisent la nouvelle norme :

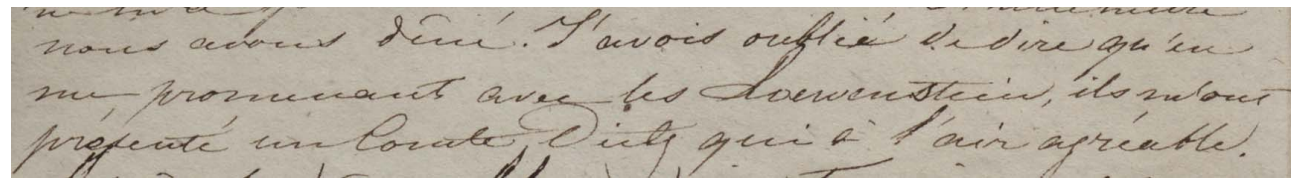

Fig. 2. Exemple d'orthographe française ancienne, J'avois oublié de dire.... (Cahier original, RGB 92-6.)

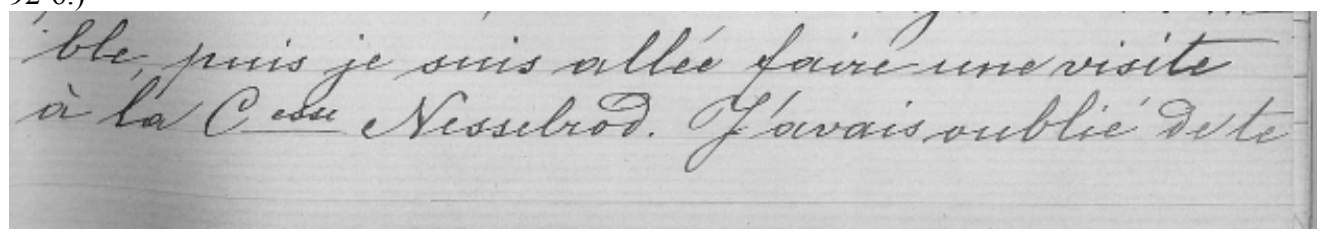

Fig. 3. Exemple d'orthographe française nouvelle, J'avais oublié de dire.... (GPNTB 601-1.)

Par ailleurs, nous n'avons pas considéré comme erronées les graphies dîné ou déjeûné pour diner et déjeuner, norme qui ne s'est fixée que récemment (pour le circonflexe) et au début du XXe siècle (pour l'infinitif substantivé). 
Les erreurs présentes dans les copies analysées sont du domaine de l'orthographe, de la grammaire et du lexique, dans l'ordre décroissant de fréquence. L'étude en cours portant sur les erreurs dans les cahiers originaux nous porte à attribuer ces erreurs, dans leur grande majorité, au copiste plutôt qu'à la diariste.

\subsubsection{Orthographe}

Les fautes d'orthographe sont les plus fréquentes dans notre corpus, on en a relevé 679, soit 822 compte tenu des répétitions. Les problèmes concernent les difficultés suivantes:

\section{A. Signes diacritiques}

Ce type d'erreur est le plus répandu, on en a dénombré 186 exemples, régulièrement répartis dans les cahiers 2-4 (37, 30 et 42). Il n'y en a que 6 sur les 80 pages en français du cinquième cahier et, par contre, presque deux fois plus dans le premier.

Les erreurs sont de quatre types (voir Tableau 2) :

- signe diacritique inutile : J'ai été intérrompue par la visite de Mr Смолинъ.

- omission du signe diacritique : J'ai fait bien des reflexions sur cette nouvelle année.

- signe diacritique présent, mais pas sur la bonne lettre : Le matin visite du medécin.

- mauvais choix du signe diacritique : Il y avait quatorze degrès de froid.

\section{B. Lettres géminées.}

Les erreurs liées aux consonnes géminées occupent en fréquence la deuxième place dans les cahiers.

De même que pour les signes diacritiques, on rencontre les situations suivantes :

- Gémination indue : J'ai eu hier un chagrin bien innattendu.

- Absence de gémination: Je suis aujourd'hui d'une humeur raisoneuse.

On trouve également des cas exceptionnels : une voyelle géminée indument dans un mot emprunté à l'allemand, langue que OIOD ne savait pas, malgré l'origine allemande de sa mère et ses fréquents voyages à Berlin et Potsdam : C'est une Seehnsucht de te voir qui la produit. (Sehnsucht) et quelques rares cas de gémination erronée (combination de gémination absente et erronée : Je reviens à la maison triste et abbatue.

Il convient par ailleurs de distinguer les erreurs des cas de notation typographique de la consonne géminée sous forme d'un trait horizontal sur la lettre : courone, home, feme, naisance etc. (fig. 4). Cet emploi n'est cependant pas systématique.

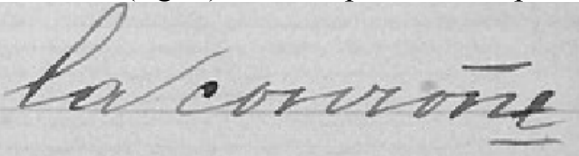

Fig. 4. Trait horizontal pour indiquer la gémination de la consonne (601-4) la couroñe.

\section{Autres cas d'orthographe lexicale \\ Lettre omise}

Il s'agit le plus souvent de lettres muettes à la fin du mot (ces cas sont presque deux fois plus fréquents qu'une omission à l'intérieur du mot) : Ce matin j'ai pris mon portefeuil et mes lettres avec moi; Dimanche chez la vielle Princesse Dolgorouky.

Un cas unique de lettre (muette) absente au début du mot: Commencement de l'iver.

\section{Lettre superflue}

Il s'agit là aussi de lettres muettes, en fin de mot, et le seul cas en initiale est un ' $h$ ' non aspiré. Ces erreurs sont réparties de manière égale dans les cahiers: Reviens auprès de celle qui t'aime commes tu crois l'aimer ; <...> composé de livre de piété fort curieux et de manuscripts $<\ldots>$; $<\ldots>$ creusée par un hermite.

\section{Lettre erronée}


Les cas rencontrées relèvent de deux situations différentes. D'une part il s'agit de problèmes d'orthographe lexicale, quand le mot est écrit phonétiquement correctement, mais pas orthographiquement : tu me croyes moins forte; des poliçoneries ; son gouâtre; Il est devenu un peu plus serain vers le soir.

Dans d'autres cas, il s'agit d'erreurs qui relèvent de l'orthographe grammaticale : le mot ainsi orthographié correspond à une forme existante du français, mais ce n'est pas celle qui est nécessaire ici. Alexandre a fait tous ces préparatifs de départ ; cela m'étais désagréable d'être seule; Je gémie et pleure.

Nous considérons qu'il s'agit ici de fautes d'orthographe: l'auteure ou son copiste savent pertinemment quelle forme on doit utiliser, ils se trompent sur sa graphie; Ces erreurs sont assez fréquentes avec l'impératif des verbes en -er, orthographié comme une deuxième personne du singulier, ou les formes des verbes en -oudre.

\section{Mots composés}

On rencontre quelques cas d'écriture en un mot de lexèmes qui doivent l'être en deux. Certaines de ces erreurs sont récurrentes, comme parceque, denouveau, delà, et peuvent dans certains cas s'expliquer par une interférence avec le russe. On peut rapprocher ces erreurs de celles qui consistent à recoller en un mot des formes comprenant une apostrophe : aller dabord dans ces vignes. D'autres semblent être de simples erreurs d'inattention comme monchant ou un cas inverse de mot coupé indument $<\ldots>$ qui en lacent ma tante.

On a relevé 42 erreurs dans l'emploi du trait d'union. Dans la plupart des cas le trait d'union est omis : la sage femme, mon beau frère, de l'eau de vie. On trouve cette erreur également dans certaines constructions grammaticales : de ce côté-là, imagine toi, rends moi. A l'inverse, dans certains cas on trouve systématiquement une graphie avec trait d'union là où il n'en faut pas : sang-sue, passe-port. Cette dernière graphie peut être admise car courante au XIXè siècle, de même tout-à-fait ou très-grand;

\section{Emploi de la majuscule.}

Les erreurs sur l'emploi des majuscules portent exclusivement sur les noms désignant une nationalité, tels italien, russe, allemand. Dans certains cas la majuscule est absente, par ex. : J'ai une conversation vive sur les russes. Plus fréquemment une majuscule avec l'adjectif qualificatif est employée à tort: Les premiers mots ont été adressés à Maman en Allemand.

Pour terminer ce petit tour d'horizon des fautes d'orthographe rencontrées dans les carnets intimes, notons quelques mots dans lesquels plusieurs types de fautes sont combinés : un fort rhume catharale; Je m'assay à reculons; un guet à pen.

Le Tableau 2 présente la répartition des fautes d'orthographe selon les cahiers. Nous tenons à souligner qu'un nombre total d'erreurs relevées de 822 sur 163259 mots (sur plus de 1000 pages) indique un très fort coefficient de correction de la langue française analysée, avec moins d'une erreur par page.

Tableau 2. Répartition des erreurs orthographiques par cahier

\begin{tabular}{|l|l|l|l|l|l|l|}
\hline & total & $601-1$ & $601-2$ & $601-3$ & $601-4$ & $601-5$ \\
\hline gémination & 137 & 45 & 27 & 15 & 36 & 14 \\
\hline signes diacritiques & 186 & 71 & 37 & 30 & 42 & 6 \\
\hline omission d'une lettre & 96 & 31 & 16 & 20 & 24 & 5 \\
\hline lettre superflus & 85 & 23 & 22 & 20 & 16 & 4 \\
\hline lettre erronée & 95 & 20 & 21 & 28 & 21 & 3 \\
\hline lettres interverties & 4 & $\mathrm{x}$ & $\mathrm{x}$ & 3 & 1 & $\mathrm{x}$ \\
\hline un/deux mots & 11 & 7 & 1 & 1 & 1 & 1 \\
\hline trait d'union & 41 & 21 & 1 & 13 & 6 & 1 \\
\hline majuscules & 19 & 2 & 4 & 3 & 7 & 3 \\
\hline
\end{tabular}




\begin{tabular}{|l|l|l|l|l|l|l|}
\hline erreurs combinées & 8 & 2 & 2 & 2 & 2 & $\mathrm{x}$ \\
\hline total des types & 679 & 222 & 130 & 135 & 156 & 36 \\
\hline total des erreurs & 822 & 324 & 161 & 152 & 150 & 36 \\
\hline
\end{tabular}

Il convient toutefois de souligner que les erreurs concernant les noms propres (anthroponymes et toponymes) n’ont pas été relevées ici. Elles ne relèvent pas à proprement parler de l'orthographe française. En ce qui concerne les noms de famille, mis à part les rares erreurs dans les noms de famille français (Choiseul systématiquement orthographié Choiseuil, par exemple), la plupart des erreurs porte sur les noms d'origine étrangère (le nom de leur intendant à Oussolié orthographié tantôt Brumer, tantôt Brummer), russe souvent: la difficulté réside alors dans le choix des règles de translittération, qui n'étaient probablement pas définitivement fixées à l'époque.

Les autres erreurs concernent les toponymes, étrangers pour la plupart. Dans la cas de petites villes ou villages traversés au cours de leurs pérégrinations, OIOD notait probablement ces noms d'oreille, sans avoir la possibilité de les vérifier par la suite, et la personne qui les a copiées ne les connaissait pas.

\subsubsection{Grammaire}

Les fautes de grammaire sont ne sont pas très variées ni fréquentes, on en a relevé 394 dont certaines récurrentes, ce qui donne un total de 562 erreurs. Par leur type elles s'apparentent beaucoup plus aux erreurs de francophone qu'à celles de russophones apprenant le français [3].

Dans les carnets analysés on trouve surtout des fautes d'accord, des constructions verbales fautives, et quelques erreurs dans l'emploi des articles et des prépositions.

\section{Accord du participe}

Les erreurs les plus fréquentes (la moitié) sont des fautes dans l'accord du participe passé, surtout quand il est employé avec l'auxiliaire avoir, ce qui n'est pas très étonnant, considérant la difficulté de la règle en question (voir infra Tableau 3).

Avec l'auxiliaire avoir, on trouve des accords superflus (avec le sujet) : toute la semaine a passée sans événements particuliers, ce qui peut être considéré comme une influence du russe, mais on trouve beaucoup plus souvent des accords manquants, ce qui est une erreur typique des francophones: J'ai reçu de retour ma lettre adressée à toi, à Moscou, tu ne saurais croire comme cela m'a contrarié.

Quant aux accords fautifs avec l'auxiliaire être, l'absence d'accord est rare : A peine descendu là que l'infatiguable Sébastiani nous fait voir tout le château, l'accord superflu est pour sa part lié au cas d'un verbe pronominal construit avec un complément d'objet indirect : Thérèse s'est imaginée <... > que je me méfiais d'elle.

Outre l'accord du participe passé dans les temps composés, on peut rencontrer des erreurs d'accord (manquant ou superflu) dans d'autres constructions verbales (par exemple au passif, J'ai été engagé à une soirée du Roi), des accords erronés du participe présent : de jolis villages, à nous appartenants, et de simples fautes d'accords de l'adjectif : sa faim et sa soif de la vérité, qui doivent être satisfaite un jour.

Dans certains cas il est difficile de juger s'il s'agit d'une faute d'orthographe lexicale ou d'une faute d'accord : des souvenirs d'amour qui étaient restés intactes dans leur mémoire. Etant donné que le participe passé restés est correctement accordé au masculin pluriel, la graphie intacte, a pu être utilisée ici pour refléter la prononciation correcte, [z̃takt], de cet adjectif.

\section{Constructions verbales fautives}

Ces erreurs ne sont pas fréquentes, et consistent principalement en la confusion du participe passé et de l'infinitif (verbes du premier groupe), par ex. J'ai laissé passé tant de jours sans 
écrire, quelques rares confusions subjonctif / indicatif, dans les deux sens : Il envoie son neveu demander au médecin, combien il lui donne à vivre, et qu'il lui dit la vérité ; La petite lampe brûle devant elle nuit et jour et qu'elle l'entretienne en travaillant.

Dans certains cas l'erreur consiste en une rection erronée, notamment celle relativement fréquente parmi tous les non-francophones du verbe aider construit avec un complément indirect : Maman peut espérer de rejoindre ses enfants de Moscou; Je voyais souffrir mon ami sans pouvoir lui aider; Калининъ nous attend à l'autre bord. Considérant les difficultés que présente le choix de la préposition en français pour les non-francophones, ces erreurs témoignent d'une très bonne maitrise de la grammaire française par OIOD, et qui plus est d'une compétence grammaticale caractéristique d'un francophone, et non d'un apprenant allophone.

Cette hypothèse est confirmée par la rareté des erreurs de concordance des temps : La chambre était remplie de monde et bientôt les 60 petites élèves entonnèrent l'hymne :"Христос рождается"<..> Après cela elles chantaient le Великое славословие extrêmement bien, ou de choix de l'auxiliaire : Ensuite nous avons descendu au Forum.

\section{Emploi des articles}

On constate un petit nombre d'erreurs dans l'emploi des articles, principalement en combinaison avec des prépositions. Si on considère que dans les autres constructions les articles sont toujours corrects, il n'y a pas de confusion entre les articles définis et indéfinis, on peut penser qu'il s'agit ici d'erreurs d'inattention : La ville du Francfurt; Il a parlé ensuite de Grand Duc Constantin; un billet pour la chambre de députés; Chez le Tchernichef.

\section{Genre et nombre}

Les erreurs portant sur le genre et le nombre des substantifs forment une catégorie à cheval entre le lexique et la grammaire : en effet, on peut considérer que le genre du substantif fait partie de ses caractéristiques, surtout en français où il n'est pas justifié par la forme du mot. Les erreurs ici sont rares, 28 en tout: On a célébré un espèce de mariage; Je donne à la Comtesse un petit livre sur la purgatoire. Quant au nombre (sans compter les fautes d'accord dues probablement à l'inattention, par ex.: J'en suis humiliée, et cette humiliations m'est nécessaire) on constate quelques erreurs récurrentes inexplicables, comme : Oтецъ Григорій nous a tenu un petit discours, qui a fait fondre en larme toute l'Eglise

\section{Accord des noms de famille au pluriel}

Ce type d'erreurs est assez fréquent, ce qui s'explique facilement par le nombre très important de noms de famille dans le texte des carnets intimes. Cependant, l'emploi est très irrégulier, même à l'intérieur d'une seule et même entrée :

Dimanche 20 avril 1847

Messe - bonne lecture avec les enfants - le soir au spectacle russe - très mauvais Levacheff - Souvoroff à côté de nous - les Anatoles avec nous. Ces trois personnages si près les uns des autres faisaient un singulier effet. - Les enfants vont chez les Zouboffune partie depuis longtemps projetée $<\ldots>(601-5)$.

Difficile à dire si l'erreur est provoquée par une interférence avec le russe, où les noms propres prennent la désinence du pluriel si nécessaire, où à une hypercorrection en français, qui demande une marque du pluriel après un déterminant pluriel.

Tableau 3. Répartition des erreurs de grammaire par cahier

\begin{tabular}{|l|l|l|l|l|l|l|}
\hline & total & $601-1$ & $601-2$ & $601-3$ & $601-4$ & $\begin{array}{l}601- \\
5\end{array}$ \\
\hline accord du participe avec avoir & 160 & 51 & 32 & 34 & 31 & 12 \\
\hline
\end{tabular}




\begin{tabular}{|l|l|l|l|l|l|l|}
\hline accord du participe avec être & 35 & 10 & 2 & 5 & 10 & 8 \\
\hline autres fautes d'accord & 27 & 7 & 2 & 4 & 11 & 3 \\
\hline constructions verbales fautives & 7 & 3 & 1 & 2 & 1 & $\mathrm{x}$ \\
\hline confusion infinitif/participe & 24 & 7 & 6 & 4 & 7 & $\mathrm{x}$ \\
\hline rection verbale fautive & 12 & 6 & 1 & 1 & 4 & $\mathrm{x}$ \\
\hline articles & 30 & 9 & 5 & 3 & 11 & 2 \\
\hline pronoms & 5 & 1 & 1 & 1 & 1 & 1 \\
\hline genre & 28 & 5 & 5 & 10 & 3 & 5 \\
\hline nombre & 56 & 8 & 8 & 13 & 22 & 5 \\
\hline pluriel des noms propres & 3 & 1 & $\mathrm{x}$ & $\mathrm{x}$ & 1 & 1 \\
\hline cas complexes & 7 & 1 & 1 & 3 & 2 & $\mathrm{x}$ \\
\hline nombre total d'erreurs & 394 & 109 & 64 & 80 & 104 & 37 \\
\hline nombre d'occurences & 562 & 153 & 93 & 115 & 161 & 40 \\
\hline
\end{tabular}

\subsubsection{Lexique}

Les erreurs lexicales ne sont pas fréquentes, et on n'en a relevé que 49. Pour certaines, on ne saurait dire s'il s'agit d'une faute d'orthographe ou d'accord due à l'inattention, par ex . jolie place du marcher ; Ce matin pluie averse ; une boite de couleur (il s'agit d'une boite de couleurs).

La plupart des erreurs peut être considérée comme des calques du russe, notamment dans les expressions: la mère assise; nous pourrons être réunies une fois et pour toujours; c'est la première fois que j'ai vu le Mitropolitain. Mme Ludger est venue me féliciter avec les fêtes.

Dans certains cas, on note des confusions de paronymes (adopter au lieu d'adapter), des quasi-synonymes (sûreté au lieu de sécurité), ou le genre du mot (la tour au lieu de le tour). Quoi qu'il en soit les erreurs sont rares. Une étude ultérieure permettra d'analyser la richesse du vocabulaire de l'auteur.

\section{Analyse des cahiers originaux}

Une analyse comparative des originaux conservés à la RGB et des copies conservées à la GPNTB nous a permis de faire les conclusions suivantes :

- Dans les premiers cahiers (rédigés vers les années 30) les erreurs de français (surtout d'accord du participe passé) sont fréquents dans les originaux, et corrigés dans la copie, cf : Les Davidofs ont dinés $\rightarrow$ ont diné; nous ne les avons pas trouvé à la maison $\rightarrow$ trouvés ; plus tard elles sont venu chez nous $\rightarrow$ venues. Cependant toutes ces erreurs ne sont pas corrigées et on en trouve dans les copies également.

- par la suite, le niveau de correction des carnets s'améliore et les erreurs que l'on relève dans les copies proviennent de l'original et ne sont pas corrigées tant dans le domaine de la grammaire que dans celui de l'orthographe lexicale, par ex: Les G[andes]. D[uches]ses m'ont frappées par leur mauvaise tenue; il a pris un air grave et digne qui lui manquait complèttement;

- Dans certains cas, le copiste n'a non seulement pas corrigé les erreurs de l'original, mais en a rajouté quelques-unes dont certaines peuvent être mises au compte de l'inattention: Nous avons marché par les chambres $\rightarrow$ marchés; Une horrible histoire $\rightarrow$ horible.

L'apparition dans la copie du hapax chiens *chargneux est due à une mauvaise lecture de l'original, où figure l'expression chiens haineux, de même *sémérité pour sévérité, lamention pour lamentation et certains autres. La phrase énigmatique Elle a emporté toutes 
les dignes presque jusqu'au cadran salaire retrouve son sens dans l'original, où il s'agit de digues et d'un cadran solaire.

Les erreurs du copiste sont plus fréquentes là où le texte est difficile à déchiffrer, par exemple en bout de ligne, où OIOD avait tendance à resserrer les lettres et descendre vers le bas. Par exemple, dans le carnet original, « recueille » se trouve en fin de ligne, et le copiste note : Il faut semer avec tristesse pour recueille avec joie. De même, le copiste n'a pas toujours bien interprété (et n'a pas fait l'effort de réfléchir au sens de ce qu'il écrivait) quand le mot manuscrit pouvait être déchiffré de plusieurs manières : soldat qui a été en Russie, aire Napoléon (au lieu de « avec »), le pauvre homme s'embarrasse tellement qu'il tombe de mal au père (au lieu de «mal en pire»), dessiner les églises qu'il remontre (rencontre) sur la route;

Une comparaison détaillée du cahier original 92-13 (notes correspondant à l'année 1844) et de sa copie, répartie dans les cahiers $601-2$ et $601-4$ a montré que sur 475 cas d'erreurs relevées dans la copie, dans 372 cas elles provenaient du copiste et seulement dans 103 cas de OIOD. Parmi ces dernières dans 76 cas le copiste n'a pas relevé ou corrigé l'erreur d' OIOD, et ne l'a fait que dans 27 cas.

On peut penser que le copiste a parfois réalisé ce travail mécaniquement. Impossible, par exemple, d'expliquer autrement l'apparition deux fois de suite dans la copie de : et je suis allée aux matinées au lieu de matines alors ce mot est fréquent dans les manuscrits (à la différence de matinée qui ne se retrouve nulle part ailleurs), Il se peut également qu'il ignore certains mots employés par OIOD, par ex. autographe (orthographié othographe dans l'original et copié abusivement orthographe) ou tribulation (copié tributation).

Un certain nombre d'erreurs relevées dans les copies font penser qu'elle s'effectuait peut-être sous la dictée : ainsi, dans l'extrait suivant : ensuite grande promenade dans le parc que je ne croyais pas aussi beau — la scène le traverse, de toute évidence il s'agit de la Seine (les Davidof se trouvent dans l'ancien parc de Neuilly). De même, des erreurs comme je me lève de bonheur ; Wladimir emparait content; qu'on nose regarder semblent découler d'une confusion à l'oral sans analyse critique de la part du copiste. Une étude ultérieure permettra de discriminer plus finement les erreurs dues à OIOD et celles qui proviennent du copiste. Quoi qu'il en soit, nous sommes définitivement en présence d'un texte d'un très haut niveau de correction, dans lequel on ne peut déceler que quelques éléments erronés dus à l'influence de la langue maternelle.

\section{Conclusions}

La francophonie russe du XIXe siècle est un vaste phénomène qui continue à être activement étudié. On se penche actuellement sur les méthodes d'enseignement de l'aristocratie russe et le rôle des percepteurs français [4]. Il ne fait aucun doute que, malgré la mode du français, les enfants de ce milieu étaient tout d'abord éduqués en russe. Voici ce qu'en dit OIOD elle-même, alors qu'elle se trouve en villégiature à Revel après trois ans passés à l'étranger, en France notamment: «Tous les enfants qui nous entourent parlent français avec un fort accent russe qui ne frappe que lorsque l'on a été habitué à entendre les enfants aux Tuileries. Quelle folie de ne parler sa propre langue »(601-4).

Par ailleurs, le russe était la langue de leur vie spirituelle - où qu'ils soient, les Davidof suivent la liturgie orthodoxe, dans des chapelles privées en cas d'impossibilité d'assister à un office dans une église orthodoxe russe.

Quant au français, on peut supposer que OIOD a commencé à l'apprendre à l'âge de quatre ans, suite à l'arrivée dans leur maison d'une gouvernante suisse : "Je suis née à Moscou le 15 Avril 1814. Papa et Maman ont beaucoup voyagé dans mon enfance, et moi j'étais toujours de leurs voyages. C'est aussi avec eux que je suis allée à Genève à l'âge de quatre ans, et c'est de là que mes parents ont amené ma gouvernante qui sortait alors de pension» (601-2). Comme indiqué en [5], après le français OIOD a étudié l'italien et 
l'anglais, mais elle ne savait pas l'allemand; Le français lui servait donc non seulement pour la communication avec les personnes de son milieu, mais également de langue de communication internationale : c'est en français qu'elle discute, par exemple, avec la police à Marienbad quand elle perd sa montre : on m'annonce le commissaire de police. - C'est un élégant, en gants blancs qui me fait en bon français des excuses de me déranger si tôt... Elle ne manque d'ailleurs jamais de railler les personnes de son rang qui le parlent mal, par exemple le diplomate du Hannovre A. Münchhausen (1813-1886): le soir arrive Münchausen, qui fait rire Alexandre par son français.

Dans cette étude nous tenions à démontrer objectivement le niveau élevé de maitrise du français de ces francophones, grâce à l'étude du "parler bilingue» spécifique de l'aristocratie russe du XIXe siècle, tel qu'il est reflété dans les carnets intimes, et aussi à l'analyse des erreurs relevées dans notre corpus. Nous nous attacherons par la suite à étudier les méthodes utilisées pour l'enseignement de cette langue, telles qu'on peut les trouver, par exemple, dans les cahiers des membres de la famille impériale.

\section{Références}

1- Gretchanaia, C. Viollet, Diaristes et épistolières russes (fin XVIIIe - début XIXe siècle) : reflets de l'histoire, Genre \& Histoire [Online], 9 | Automne 2011, http://genrehistoire.revues.org/1396

2- Gretchanaia, "Je vous parlerai la langue de l'Europe...»: La francophonie en Russie $\left(X_{\text {XIII }}^{\mathrm{e}}-\mathrm{XIX}{ }^{\mathrm{e}}\right.$ siècles), Bruxelles - Berlin - New York - Oxford - Vienne : Peter Lang, 2012, $411 \mathrm{p}$.

3- Gretchanaia, C. Viollet et al. «Si tu lis jamais ce journal...»: Diaristes russes francophones, 1780-1854. Paris: CNRS-Editions, 2008

4- V. Rjéoutski Quand le français gouvernait la Russie. L'éducation de la noblesse russe 1750-1880 Paris, Editions L'Harmattan, 402 p.

5- Les erreurs de français des russophones. Limoges: Lambert-Lucas, DL, 2011. Vol. 1. $165 \mathrm{p}$.

6- M. Debrenne The French Language in the Diaries of Olga Davydova. An example of Russian-French Aristocratic Bilingualism // Le français, langue de l'intime à l'époque moderne et contemporaine. Edited by Madeleine van Strien-Chardonneau and MarieChristine Kok Escalle, Amsterdam, Amsterdam University Press B.V., 2016, 125-142 